\title{
Natural vibrations of nanostrips with cracks
}

\author{
Mainul Hossain and JaAn LeLleP
}

\begin{abstract}
Employing the main equations of the theory of plates accounting for the rotational inertia the transverse vibrations of nanobeams and nanostrips are investigated. The nano strips under consideration have piecewise constant dimensions of cross sections. The nanosheets are weakened by cracks at re-entrant corners of steps. While the material behavior corresponds to the Eringen's nonlocal theory of elasticity it is assumed that the cracks produce additional local compliance, which can be evaluated with the aid of the stress intensity factor at the crack tip. A numerical algorithm for determination of natural frequencies of nanosheets is developed.
\end{abstract}

\section{Introduction}

The analysis of nano-sized structural elements has shown that the behavior of nanobeams and nanoplates can be modelled with the aid of sizedependent theories only. The classical theory of elasticity is not suitable for this purpose as it is unable to account for the size effects. A suitable nonlocal theory of elasticity was developed by Eringen [10], also Eringen and Edelen [11]. According to Eringen [10] the stress at a fixed point of the body depends on the strain field at each point of the body.

Peddieson et al. [23] applied the nonlocal theory of elasticity for investigation of actuators in small-scale systems. Later Reddy [25] captured the modifications of the Euler-Bernoulli, also Timoshenko and Reddy beam theories into a non-local beam theory resorting to the differential constitutive relations of Eringen. Analytical solutions of bending, vibration and buckling are developed for nanomaterials with differential constitutive equations by Reddy [25], Bagdatli [6], Ansari and Sahmani [3], Lu et al. [21], Ghannadpour et al. [13], Wang et al. [30, 31], Thai [29], Li et al. [19], Aranda-Ruiz et al. [4], Zhou and Huang [35], Hossain and Lellep [14],

Received September 11, 2020.

2020 Mathematics Subject Classification. 74B20.

Key words and phrases. Nano material, natural vibrations, crack, step, rotatory inertia. https://doi.org/10.12697/ACUTM.2021.25.06 
Lim et al. [20], Zhang et al. [34]. While the papers cited above use the Eringen's nonlocal theory with differential constitutive equation FernándezSáez et al. [12] formulated the problem making use of the Eringen's integral constitutive equation. Roostai and Haghpanahi [26] investigated the free vibration of nanobeams with multiple cracks. The effects of nonlocality, crack location and crack parameter are examined in case of natural frequencies of the cracked nanobeam.

Aydogdu [5] presented a nonlocal beam theory for studying bending, buckling and vibration problems of nanobeams. This approach admits to employ the beam theories including those of Euler-Bernoulli, Timoshenko, Reddy, Levinson and Aydogdu for solution of particular problems. Variational principles regarding to the complementary energy and potential energy, also $\mathrm{Hu}$-Washizu principles are connected with the concept of nonlocal elasticity by Polizzotto [24]. The variational approach to solution of problems of strain gradient elasticity was employed by Challamel and Wang [7], as well.

Mousavi et al. [22] presented a variational approach to the analysis of third-order shear deformable plates. Higher order shear deformation beams are investigated within the strain gradient theory by Yaghoubi et al. [33]. All the papers deal with the natural vibrations. Moreover, the industrial problem of the material damping is often so important that it cannot be neglected. Wesolowski and Barkanov [32] developed a new method for identification of coefficients of material damping for laminated plates.

In the present paper natural vibrations of nanobeams are investigated in the case of step-wise varying cross sections. It is assumed that the nanobeams are weakened by stable cracks at the re-entrant corners of steps.

\section{Statement of the problem}

Consider the natural vibrations of a nanoplate strip of length $l$ with rectangular cross section of width $b$ and height

$$
h= \begin{cases}h_{0}, & \mathrm{X} \in(0, a), \\ h_{1}, & \mathrm{X} \in(a, l),\end{cases}
$$

where $h_{0}, h_{1}$ are given constants.

It is assumed that the coordinate axis $0 X$ coincides with the axis of the straight nanoplate strip and its origin is located at the center of the lefthand edge of the nanoplate. The nanoplate strip is stretched with the axial load $N$. This nanoplate of piecewise constant thickness (height of the cross section) has defects at the cross section $X=a$. The defects are treated herein as stable cracks of length $c$. The stability of the crack is meant in the present analysis so that the crack does not propagate during the vibration with fixed amplitude. However, it is accepted that the presence of the crack causes the change of the internal (potential) energy of the nanoplate strip. 


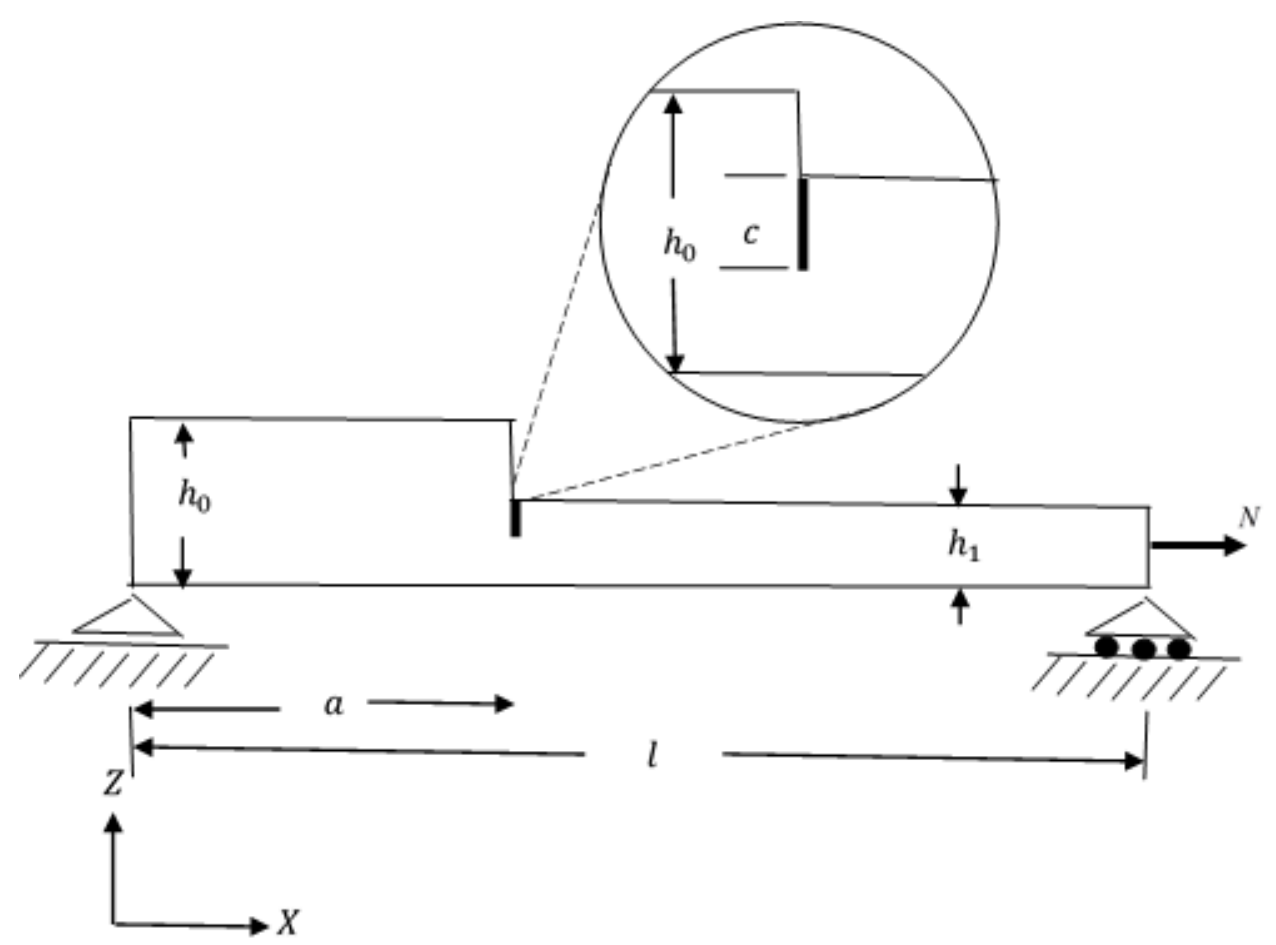

Figure 1. Geometry of the problem

The aim of the paper is to determine the frequencies of natural vibrations of a nanoplate strip simply supported and free of external loads. Moreover, we are aiming at the definition of the sensitivity of eigenfrequencies on the geometrical parameters of the nanoplate. The primary concern of the study is to elucidate the dependence of eigenfrequencies on the crack location $a$, its length $c$ and dimensions $b, h_{0}, h_{1}$.

\section{Governing equations}

The governing equations of a vibration problem consist of geometrical relations, equilibrium equations and constitutive equations with boundary conditions. A geometrically non-linear beam model accounting for the effect of the inertia of rotation will be used in the present study. Assuming that the axial force $N=$ const. throughout the nanobeam one can present the equilibrium equations as (see Reddy [25], Soedel [27])

$$
\frac{\partial^{2} M}{\partial X^{2}}+N \frac{\partial^{2} W}{\partial X^{2}}=\mu \frac{\partial^{2} W}{\partial t^{2}}-I_{r} \frac{\partial^{4} W}{\partial X^{2} \partial t^{2}} .
$$


Here $M(X, t)$ denotes the bending moment, $W(X, t)$ the transverse displacement of a point lying on the axis of the nanoplate strip, and $t$ is time. The parameter $\mu$ in (2) stands for the mass per unit length of the nanobeam and $I_{r}$ is the moment inertia of the plate strip.

Since the height of the nanoplate strip is according to (1) piece wise constant it is reasonable to introduce the notations $I_{r}=\mu I$, where

$$
\mu= \begin{cases}\mu_{0}, & \mathrm{X} \in(0, a), \\ \mu_{1}, & \mathrm{X} \in(a, l)\end{cases}
$$

and

$$
I= \begin{cases}I_{0}, & \mathrm{X} \in(0, a), \\ I_{1}, & \mathrm{X} \in(a, l) .\end{cases}
$$

In (3) for homogeneous beam $\mu_{0}=\mu_{1}=\rho$ where $\rho$ is the density of the material. The inertial moments $I_{0}, I_{1}$ can be calculated as

$$
I_{0}=\left(b h_{0}^{3}\right) / 12, \quad I_{1}=\left(b h_{1}^{3}\right) / 12 .
$$

Evidently, in the case $I_{r}=0$ the equilibrium equation (2) coincides with the classical non-linear equation of beam dynamics (see Reddy [25], Li et al. [19], Soedel [27], Lellep and Lenbaum [17], Lellep and Liyvapuu [18]).

It is expected that the mechanical behavior of a nanoplate strip can be prescribed within the limits of the nonlocal theory of elasticity. Probably the simpliest case of the constitutive equation was treated by Reddy [25], Lu et al. [21], Aranda-Ruiz et al. [4], Roostai and Haghpanahi [26], Lellep and Lenbaum [16, 17].

In the classical Euler-Bernoulli theory (see Lellep and Kägo [15], Lellep and Lenbaum [17], Soedel [27])

$$
M_{c}=-E I \frac{\partial^{2} W}{\partial X^{2}},
$$

where $E$ stands for the Young modulus and area moment of inertia $I$ is defined by (4) and (5). However, in the non-local elasticity one can expect that (see Roostai and Haghpanahi [26], Reddy [25], Lellep and Lanbaum $[17])$

$$
M-\left(e_{0} a\right)^{2} \frac{\partial^{2} M}{\partial X^{2}}=-E I \frac{\partial^{2} W}{\partial X^{2}} .
$$

Combining the equations (2), (6), and (7) one obtains

$$
M=-E I \frac{\partial^{2} W}{\partial X^{2}}+\left(e_{0} a\right)^{2}\left(-N \frac{\partial^{2} W}{\partial X^{2}}+\mu \frac{\partial^{2} W}{\partial t^{2}}-\mu I \frac{\partial^{4} W}{\partial t^{2} \partial X^{2}}\right)
$$


provided $\mu=$ const, $E=$ const. Substituting (8) in (2) leads to the equation

$$
\begin{aligned}
& E I \frac{\partial^{4} W}{\partial X^{4}}-\left(e_{0} a\right)^{2}\left(-N \frac{\partial^{4} W}{\partial X^{4}}+\mu \frac{\partial^{4} W}{\partial t^{2} \partial X^{2}}-\mu I \frac{\partial^{6} W}{\partial t^{2} \partial X^{4}}\right) \\
& =N \frac{\partial^{2} W}{\partial X^{2}}-\mu \frac{\partial^{2} W}{\partial t^{2}}+\mu I \frac{\partial^{4} W}{\partial t^{2} \partial X^{2}} .
\end{aligned}
$$

Separating variables in (9) one can assume that

$$
W=\bar{W}(X) \cdot T(t),
$$

where $\bar{W}(X)$ depends on $X$ only and $T(t)$ is a function of time $t$. Making use of (9) and (10), one obtains

$$
\frac{\frac{d^{2} T}{d t^{2}}}{T}=-\frac{E I \frac{d^{4} \bar{W}}{d X^{4}}+\left(e_{0} a\right)^{2} N \frac{d^{4} \bar{W}}{d X^{4}}-\left(e_{0} a\right)^{2} \mu \frac{d^{2} \bar{W}}{d X^{2}}-N \frac{d^{2} \bar{W}}{d X^{2}}}{\left(e_{0} a\right)^{2} I \mu \frac{d^{4} \bar{W}}{d X^{4}}+\mu \bar{W}-I \mu \frac{d^{2} \bar{W}}{d X^{2}}}=-\omega_{c}^{2},
$$

where $\omega_{c}$ stands for the frequency of free vibrations. The equations (9)-(11) admit to present the equation for determination of $\bar{W}(X)$ as

$$
\begin{gathered}
E I \frac{d^{4} \bar{W}}{d X^{4}}+\left(e_{0} a\right)^{2}\left(N \frac{d^{4} \bar{W}}{d X^{4}}-\mu \frac{d^{2} \bar{W}}{d X^{2}}-\omega_{c}^{2} I \mu \frac{d^{4} \bar{W}}{d X^{4}}\right) \\
-N \frac{d^{2} \bar{W}}{d X^{2}}-\omega_{c}^{2} \mu \bar{W}+I \mu \omega_{c}^{2} \frac{d^{4} \bar{W}}{d X^{4}}=0 .
\end{gathered}
$$

The dimensionless variables are defined as follows:

$$
\begin{gathered}
x=\frac{X}{l}, w=\frac{\bar{W}}{l}, \eta=\frac{e_{0} a}{l}, n=\frac{N l^{2}}{E I}, \\
\omega_{0}=\omega_{c} l^{2} \sqrt{\frac{\rho}{E I}}, \gamma=\frac{h_{1}}{h_{0}}, \omega_{1}=\gamma \omega_{0} .
\end{gathered}
$$

Then, the equation of motion is simplified in the form

$$
\frac{d^{4} w}{d x^{4}}\left(1+\eta^{2} n-\frac{h_{0}^{2}}{12 l^{2}} \omega_{0}^{2}\right)+\frac{d^{2} w}{d x^{2}}\left(\eta^{2} \omega_{0}^{2}-n+\frac{h_{0}^{2}}{12 l^{2}} \omega_{0}^{2}\right)-\omega_{0}^{2} w=0 .
$$

For solving the linear differential equation of the fourth order (12) one has to solve the characteristic equations

$$
\left(1+\eta^{2} n-\frac{h_{0}^{2}}{12 l^{2}} \omega_{0}^{2}\right) \lambda_{0}^{4}-\lambda_{0}^{2}\left(\eta^{2} \omega_{0}^{2}-n+\frac{h_{0}^{2}}{12 l^{2}} \omega_{0}^{2}\right)-\omega_{0}^{2}=0
$$

and

$$
\left(1+\eta^{2} n-\frac{h_{0}^{2}}{12 l^{2}} \omega_{1}^{2}\right) \lambda_{1}^{4}-\lambda_{1}^{2}\left(\eta^{2} \omega_{1}^{2}-n+\frac{h_{0}^{2}}{12 l^{2}} \omega_{1}^{2}\right)-\omega_{1}^{2}=0 .
$$


It should be remembered that (13) corresponds to the solution of (12) in the interval $x \epsilon(0, a)$ and (14) for $x \epsilon(a, l)$. It is easy to recheck that the roots of (13) and (14) are

$$
\begin{aligned}
& \left(\lambda_{0}\right)_{1,2}=i \theta_{0}, \quad\left(\lambda_{0}\right)_{3,4}=\beta_{0}, \\
& \left(\lambda_{1}\right)_{1,2}=i \theta_{1}, \quad\left(\lambda_{1}\right)_{3,4}=\beta_{1},
\end{aligned}
$$

where $i$ stands for the imaginary unit, and for the conciseness sake the notation

$$
\begin{gathered}
P_{0}=\sqrt{\left(\eta^{2} \omega_{0}^{2}-n+\frac{h_{0}^{2}}{12 l^{2}} \omega_{0}^{2}\right)^{2}+4 \omega_{0}^{2}\left(1+\eta^{2} n-\frac{h_{0}^{2}}{12 l^{2}} \omega_{0}^{2}\right)}, \\
Q_{0}=\eta^{2} \omega_{0}^{2}-n+\frac{h_{0}^{2}}{12 l^{2}} \omega_{0}^{2}, \quad R_{0}=2\left(1+\eta^{2} n-\frac{h_{0}^{2}}{12 l^{2}} \omega_{0}^{2}\right), \\
\theta_{0}=\sqrt{\frac{P_{0}+Q_{0}}{R_{0}}}, \quad \beta_{0}=\sqrt{\frac{P_{0}-Q_{0}}{R_{0}}}
\end{gathered}
$$

and

$$
\begin{gathered}
P_{1}=\sqrt{\left(\eta^{2} \omega_{1}^{2}-n+\frac{h_{0}^{2}}{12 l^{2}} \omega_{1}^{2}\right)^{2}+4 \omega_{1}^{2}\left(1+\eta^{2} n-\frac{h_{0}^{2}}{12 l^{2}} \omega_{1}^{2}\right)}, \\
Q_{1}=\eta^{2} \omega_{1}^{2}-n+\frac{h_{0}^{2}}{12 l^{2}} \omega_{1}^{2}, \quad R_{1}=2\left(1+\eta^{2} n-\frac{h_{0}^{2}}{12 l^{2}} \omega_{1}^{2}\right) \\
\theta_{1}=\sqrt{\frac{P_{1}+Q_{1}}{R_{1}}}, \quad \beta_{1}=\sqrt{\frac{P_{1}-Q_{1}}{R_{1}}}
\end{gathered}
$$

is used.

The general solution of the equation of vibration (12) can be presented for $x \epsilon(0, a)$ as

$$
w=A_{1} \sinh \left(\theta_{0} x\right)+A_{2} \cosh \left(\theta_{0} x\right)+A_{3} \sin \left(\beta_{0} x\right)+A_{4} \cos \left(\beta_{0} x\right),
$$

and for $x \epsilon(a, l)$ as

$$
w=B_{1} \sinh \left(\theta_{1} x\right)+B_{2} \cosh \left(\theta_{1} x\right)+B_{3} \sin \left(\beta_{1} x\right)+B_{4} \cos \left(\beta_{1} x\right) .
$$

Arbitrary constants $A_{1}-A_{4}, B_{1}-B_{4}$ in (21) and (22) will be defined with the help of corresponding boundary and intermediate requirements. In the case of a simply supported edge the transverse displacement $W$ and the bending moment $M$ must vanish at the ends of the nanostrips.

The latter means that according to (12) at a simply supported edge

$$
\left(1+\eta^{2} n\right) \frac{d^{4} w}{d x^{4}}+\left(\eta^{2} \omega_{0}^{2}-n\right) \frac{d^{2} w}{d x^{2}}-\omega_{0}^{2} w=0 .
$$


It can be seen from (17) that at the cross sections where $w=0$ also the second derivative $\frac{d^{2} w}{d x^{2}}=0$. Thus, in the case of a nanobeam simply supported at both ends one has

$$
w(0)=0, \quad \frac{d^{2} w}{d x^{2}}(0)=0
$$

and

$$
w(l)=0, \quad \frac{d^{2} w}{d x^{2}}(l)=0
$$

Similarly, in the case of a fully clamped nanobeam the boundary conditions have the forms

and

$$
w(0)=0, \quad \frac{d w}{d x}(0)=0
$$

$$
w(l)=0, \quad \frac{d w}{d x}(l)=0 .
$$

\section{Additional local flexibility}

It is well known in solid mechanics and fracture mechanics that cracks in structures are the sources of additional flexibility or compliance $C$. In general, every displacement $u_{i}$ of the system is affected by every force $P_{j}$ applied to the system. It is known in the mechanics that the compliance $C_{i j}$ of a beam element is coupled with the applied load $P_{j}$ and the displacement $u_{i}$ as (see $\left.[14,15-18]\right)$

or

$$
C_{i j}=\frac{\partial u_{i}}{\partial P_{j}}
$$

$$
C_{i j}=\frac{\partial^{2}}{\partial P_{i} \partial P_{j}} b \int_{0}^{c} G d c .
$$

In (23), $G$ stands for the energy release rate due to the crack. On the other hand (see Dimarogonas [8], Anderson [1])

$$
G=\frac{1}{E}\left(\left(\sum_{i=1}^{6} K_{1 i}\right)^{2}+\left(\sum_{i=1}^{6} K_{2 i}\right)^{2}+(1+\nu)\left(\sum_{i=1}^{6} K_{2 i}\right)^{2}\right)
$$

In (24) $E$ and $\nu$ are the Young modulus and Poisson ratio, respectively, and $K_{1 i}, K_{2 i}, K_{3 i}$ stand for the stress intensity factors for the three modes of fracture caused by the loads $P_{i}$.

However, the problem can be remarkably simplified as most of the generalized forces $P_{i}(i=1, \ldots, 6)$ can be neglected in the case of free vibrations of the beam or plate strip. The only non-zero generalized forces in $(22)-(24)$ are the bending moment $M$ and the membrane force $N$. Moreover, the dominating member is the bending moment $M$. Thus, one can present the rotation angle of the cross section as $\theta=C . M(a, t)$. Naturally, the matrix 
$C$ is also a function of the coordinate $X$. In the case of a beam element subjected to the couple $M$ the energy release rate $G$ and the stress intensity factor $K$ are coupled as (see Anderson [1])

$$
G=\frac{K^{2}}{E^{\prime}}
$$

In (25), $E^{\prime}=E$ in the case of plane stress state. However, in the case of plane strain one has $E^{\prime}=E /\left(1-\nu^{2}\right)$.

Let the angle $\theta$ presents the jump of the slope of the deflection, or

$$
\theta=\frac{d W}{d X}(a+0, t)-\frac{d W}{d X}(a-0, t)
$$

where the quantity $\frac{d W}{d X}$ is discontinuous. It is worthwhile to mention that according to the vibration model of Dimarogonas [8] the displacement $W$ is continuous but its derivative $\frac{d W}{d X}$ is discontinuous.

It is known in the fracture mechanics that in the case of a beam element

$$
G=\frac{M^{2}}{2 b} \frac{d C}{d c}
$$

where $M=M(a, t)$, and the stress intensity factor

$$
K=\frac{6 M}{b h^{2}} \sqrt{\pi c \quad F\left(\frac{c}{h}\right)}
$$

It follows from $(22)-(28)$ that

$$
C=\frac{72 \pi\left(1-\mu^{2}\right)}{E b h_{0}^{2}} f(s),
$$

where $s=c / h$ and

$$
f(s)=\int_{0}^{c} G d c .
$$

The shape function $F(s)$ in (29) should be determined experimentally. Making use of the experimental database from the book by Tada et al. [28] one can state that

$$
F(s)=1.93-3.07(s)+14.53(s)^{2}-25.11(s)^{3}+25.80(s)^{4}
$$

or

$$
F(s)=\sqrt{\frac{2}{\pi s} \tan \frac{\pi s}{2}} \frac{0.923+2.025+0.37\left(1-\sin \left(\frac{\pi s}{2}\right)\right)^{4}}{\cos \frac{\pi s}{2}} .
$$

Note that (31), (32) can be applied in the case when the cracked beam element is loaded with the bending moment $M$. However, if the element is loaded with the moment $M$ and the tensile force $N$, then the stress intensity factor can be presented as (see Zhou and Huang [35], Anderson [1])

$$
K=\frac{\sqrt{\pi s}}{6 \sqrt{h}}\left(N F_{N}(s)+\frac{6 M}{h} F_{M}(s)\right) .
$$


In (29) as well as in (33) instead of $h$ one has to choose the smallest of the thicknesses $h_{0}, h_{1}$. Thus here

$$
h=\min \left(h_{0}, h_{1}\right) .
$$

The functions $F_{N}$ and $F_{M}$ should be determined experimentally. However, there exists a suitable database in the appropriate literature, as well. According to Tada et al. [28] one can use (32) as $F_{M}(s)$ and

$$
F_{N}(s)=\sqrt{\frac{2}{\pi s} \tan \frac{\pi s}{2}} \frac{0.752+0.199\left(1-\sin \frac{\pi s}{2}\right)^{3}}{\cos \frac{\pi s}{2}} .
$$

Similar terms of the flexibility matrix have been used by Dimarogonas et al. [9], also by Anifantis and Dimarogonas [2]. However, Zhou and Huang [35] used the correction factors in the form

$$
F_{M}=1.12-1.4(s)+7.33(s)^{2}-13.08(s)^{3}+14(s)^{4}
$$

and

$$
F_{N}=1.12-0.23(s)+10.55(s)^{2}-21.72(s)^{3}+30.79(s)^{4} .
$$

The injured cross section of the nanobeam is affected by the generalized forces $N$ and $M$. Corresponding generalized displacements are the axial displacement $u$ and the slope of the deflection $\theta=\left[\frac{d w}{d x}(a)\right]$ defined by (26). Now the quantity $C$ in (29) must be interpreted as the flexibility matrix (see Anifantis and Dimarogonas [2]). However, $\theta$ and $M$ have now the role of a generalized displacement and a generalized force, respectively. Let the flexibility (compliance) matrix be the matrix

$$
[C]=\left[\begin{array}{ll}
C_{N N} & C_{N M} \\
C_{M N} & C_{M M}
\end{array}\right]
$$

where due to the symmetry $C_{M N}=C_{N M}$. In the matrix (38)

$$
\begin{aligned}
C_{N N} & =\frac{2}{E b} \int_{0}^{c} s F_{N}^{2} d s, \\
C_{M N} & =\frac{2}{E b} \int_{0}^{c} s F_{M} F_{N} d s, \\
C_{M M} & =\frac{2}{E b} \int_{0}^{c} s F_{M}^{2} d s .
\end{aligned}
$$

The jump of the slope (25) can be defined according to (23) and (39) as

$$
\theta=N \cdot C_{M N}+M \cdot C_{M M} .
$$

Evidently, similar estimation can be derived for the axial displacement $u$ also. 


\section{Determination of natural frequencies}

For determination of natural frequencies of the nanobeam one has to determine the unknown constants in the expressions of displacements (15), (16). For this purpose the boundary conditions (18) and (19) together with intermediate continuity and jump conditions will be employed. From physical considerations it is evident that the transverse deflection $w$ and bending moment $M$ vanish at the edges $x=0$ and $x=l$ of the plate strip. Thus, it follows from (12), (15), and (16) that

$$
\begin{aligned}
A_{2}+A_{4} & =0, \\
\theta_{0}^{2} A_{2}-\beta_{0}^{2} A_{4} & =0
\end{aligned}
$$

and

$$
\begin{aligned}
B_{1} \sinh \left(\theta_{1} l\right)+B_{2} \cosh \left(\theta_{1} l\right)+B_{3} \sin \left(\beta_{1} l\right)+B_{4} \cos \left(\beta_{1} l\right) & =0, \\
\theta_{1}^{2}\left(B_{1} \sinh \theta_{1} l+B_{2} \cosh \theta_{1} l\right)-\beta_{1}^{2}\left(B_{3} \sin \beta_{1} l+B_{4} \cos \beta_{1} l\right) & =0 .
\end{aligned}
$$

It immediately follows from (41) that

$$
A_{2}=A_{4}=0
$$

and

$$
\begin{aligned}
B_{1} \sinh \theta_{1} l+B_{2} \cosh \theta_{1} l & =0, \\
B_{3} \sin \beta_{1} l+B_{4} \cos \beta_{1} l & =0 .
\end{aligned}
$$

Thus

$$
\begin{aligned}
& B_{1}=-B_{2} \operatorname{coth} \theta_{1} l, \\
& B_{3}=-B_{4} \cot \beta_{1} l .
\end{aligned}
$$

The jump condition can be presented in the form

$$
\left[\frac{d w}{d x}(a)\right]=0 .
$$

Here the square brackets stand for the difference of corresponding right and left hand limits of the slope $\frac{d w}{d x}(a)$. The quantity $\theta$ in (41) must be evaluated by (35), (8) and (14). The quantities $C_{M N}, C_{M M}$ are defined by (34) with (31), (32). Making use of (15), (16), and (42)-(46) one can present the condition of continuity of the transverse deflection at $x=a$ as

$$
\begin{aligned}
A_{1} \sinh \left(\theta_{0} a\right) & +A_{3} \sin \left(\beta_{0} a\right)-B_{1} \cos \left(\beta_{1} l\right) \sinh \left(\theta_{1}(l-a)\right) \\
& -B_{3} \cosh \left(\theta_{1} l\right) \sin \left(\beta_{1}(l-a)\right)=0 .
\end{aligned}
$$

The jump condition (41) with (15), (16), (39), and (40) reads as

$$
\begin{aligned}
A_{1} \sinh \left(\theta_{0} a\right) \theta_{0}^{2} & -A_{3} \sin \left(\beta_{0} a\right) \beta_{0}^{2}-B_{1} \cos \left(\beta_{1} l\right) \sinh \left(\theta_{0}(l-a)\right) \theta_{0}^{2} \\
& +B_{3} \cosh \left(\theta_{1} l\right) \sin \left(\beta_{1}(l-a)\right) \beta_{1}^{2}=0,
\end{aligned}
$$


where the constant $\theta$ is evaluated by (35). The continuity of the bending moment $M$ and the shear force $Q=\frac{\partial M}{\partial x}$ furnishes the equations

$$
\begin{aligned}
& -A_{1} C \sinh \left(\theta_{0} a\right) \theta_{0}^{2}+A_{3} C \sin \left(\beta_{0} a\right) \beta_{0}^{2}-B_{1} \cos \left(\beta_{1} l\right) \cosh \left(\theta_{1}(l-a)\right) \theta_{1} \\
& -B_{3} \cosh \left(\theta_{1} l\right) \cos \left(\beta_{1}(l-a)\right) \beta_{1}-A_{1} \cosh \left(\theta_{0} a\right) \theta_{0}-A_{3} \cos \left(\beta_{0} a\right) \beta_{0}=0
\end{aligned}
$$

and

$$
\begin{aligned}
& A_{1} \cosh \left(\theta_{0} a\right) \theta_{0} \omega_{0}^{2} \eta^{2}+A_{3} \cos \left(\beta_{0} a\right) \beta_{0} \omega_{0}^{2} \eta^{2}+B_{1} \cos \left(\beta_{1} l\right) \cosh \left(\theta_{1}(l-a)\right) \theta_{1} \omega_{1}^{2} \eta^{2} \\
& \quad+B_{3} \cosh \left(\theta_{1} l\right) \cos \left(\beta_{1}(l-a)\right) \beta_{1} \omega_{1}^{2} \eta^{2}+A_{1} \cosh \left(\theta_{0} a\right) \theta_{0}^{3}-A_{3} \cos \left(\beta_{0} a\right) \beta_{0}^{3} \\
& \quad+B_{1} \cos \left(\beta_{1} l\right) \cosh \left(\theta_{1}(l-a)\right) \theta_{1}^{3}-B_{3} \cosh \left(\theta_{0} l\right) \cos \left(\beta_{1}(l-a)\right) \beta_{1}^{3}=0 .
\end{aligned}
$$

The system $(43)-(46)$ is a linear homogeneous system with respect to

unknown constants $A_{2}, A_{4}, B_{2}, B_{4}$. Such system has non-trivial solutions only in the case if its determinant $\Delta$ vanishes. The equation $\Delta=0$ is solved numerically with the computer software Maple. Before that one has to calculate the integrals (34) and substitute $\theta$ from (35) in (42). It appeared that a reasonable approximation of the solution can be obtained by ignoring the term $C_{M N}$ in (35). This approach was used in the case of beams in papers by the first author [14], as well. In this case it follows from (8),(14), and (35)-(40) that $\theta=C_{N M} M(a)$ and

$$
\begin{aligned}
M(a)= & A_{1} \sinh \left(\theta_{0} a\right) \theta_{0}^{2}-A_{3} \sin \left(\beta_{0} a\right) \beta_{0}^{2}-B_{1} \cos \left(\beta_{1} l\right) \sinh \left(\theta_{1}(l-a)\right) \theta_{1}^{2} \\
& +B_{3} \cosh \left(\theta_{1} l\right) \sin \left(\beta_{1}(l-a)\right) \beta_{1}^{2} .
\end{aligned}
$$

\section{Numerical results}

The results of calculations are presented in Table 1 and Figures 2-8. These results correspond to nanosheets of length $l=1 \mathrm{~nm}$ and width $b=$ $1 \mathrm{~nm}$, height $h_{0}=0.1 \mathrm{~nm}$. The material constants are: modulus of elasticity $E=160 G P a$, Poisson ratio $\nu=0.3$. Table 1 illustrates the natural frequency for different values of nonlocal parameter, height to length ratio and mode of frequency for a simply supported nanoplate strip. In this case, the natural frequency decreases with the increase of nonlocal parameter and height to length ratio. Increase of height to length ratio increases the effect of rotatory inertia. That is why, increase of height length ratio decreases natural frequency. The effect of rotatory inertia is more significant in the case of higher modes of frequency. It can be seen from Table 1 that the results of the current paper differ slightly from those obtained by Roostai and Haghpanahi [26] in the case of nanostrips of constant thickness. In paper [26], the rotatory inertia is ignored. However, the discrepancies between these results are more obvious in the case of a higher mode of frequency. 


\begin{tabular}{|c|c|c|c|c|c|c|}
\hline \multicolumn{2}{|c|}{} & $\begin{array}{c}\text { without rotatory } \\
\text { inertia }\end{array}$ & \multicolumn{4}{|c|}{$\begin{array}{c}\text { with rotatory } \\
\text { inertia }\end{array}$} \\
\hline & & H. Roostai $[19]$ & $h / l=0.05$ & $h / l=0.1$ & $h / l=0.2$ & $h / l=0.3$ \\
\hline Mode & $\eta$ & $\omega$ & $\omega$ & $\omega$ & $\omega$ & $\omega$ \\
\hline 1 & 0 & 9.8700 & 9.8590 & 9.8297 & 9.7105 & 9.5239 \\
2 & 0 & 39.4780 & 39.3174 & 38.8471 & 37.1123 & 34.6778 \\
3 & 0 & 88.8260 & 88.0139 & 85.7089 & 78.0239 & 68.8143 \\
1 & 0.5 & 5.3000 & 5.2947 & 5.27854 & 5.2155 & 5.1140 \\
2 & 0.5 & 11.9740 & 11.9260 & 11.7820 & 11.2565 & 10.5186 \\
3 & 0.5 & 18.4390 & 18.2696 & 17.7932 & 16.1972 & 14.2841 \\
1 & 1 & 2.9940 & 2.9904 & 2.9812 & 2.9455 & 2.8887 \\
2 & 1 & 6.2050 & 6.1799 & 6.1054 & 5.8335 & 5.4501 \\
3 & 1 & 9.3720 & 9.2872 & 9.0432 & 8.2319 & 7.2603 \\
1 & 1.5 & 2.0490 & 2.0462 & 2.0404 & 2.0159 & 1.9768 \\
2 & 1.5 & 4.1650 & 4.1485 & 4.0986 & 3.9156 & 3.6585 \\
3 & 1.5 & 6.2680 & 6.2105 & 6.0473 & 5.5055 & 4.8557 \\
1 & 2 & & 1.5495 & 1.5449 & 1.5264 & 1.4969 \\
2 & 2 & & 3.1186 & 3.0815 & 2.9437 & 2.7508 \\
3 & 2 & & 4.6627 & 4.5408 & 4.1335 & 3.6453 \\
\hline
\end{tabular}

TABLE 1. Natural frequency $(\omega)$ of a simply supported nanoplate strip for different values of height to length ratio $\left(\frac{h}{l}\right)$.

In Figures 2-8, the natural frequencies of stepped nanosheets are presented in the case of simply supported one-stepped nanosheets. The dashed lines correspond to the theory neglecting the rotatory inertia and the solid lines present the results of the study accounting for the effect of rotatory inertia. It can be seen from Figures $2-8$ that the bending theory accounting for rotatory inertia yields the results which essentially differ from the results obtained by ignoring the effect.

In Figure 2 the natural frequency versus nonlocal parameter is presented for different lengths of the strip. This graph shows the comparison between current study and the study of Roostai and Haghpanahi [26]. It can be seen from Figure 2 that longer nanostrips have higher natural frequencies, as might be expected. Increase of length decreases the effect of nonlocal parameter where $\eta=\frac{e_{0} a}{l}$. That is why, frequency decreases for the increase of length. The upper pair of curves in Figure 2 corresponds to a nanostrip with length $l=10 \mathrm{~nm}$; the lower one is associated with length $l=1 \mathrm{~nm}$; the middle pair corresponds to the case of length $l=5 \mathrm{~nm}$. The results of the current study compare favorably with the results of Roostai and Haghpanahi $[26]$. 


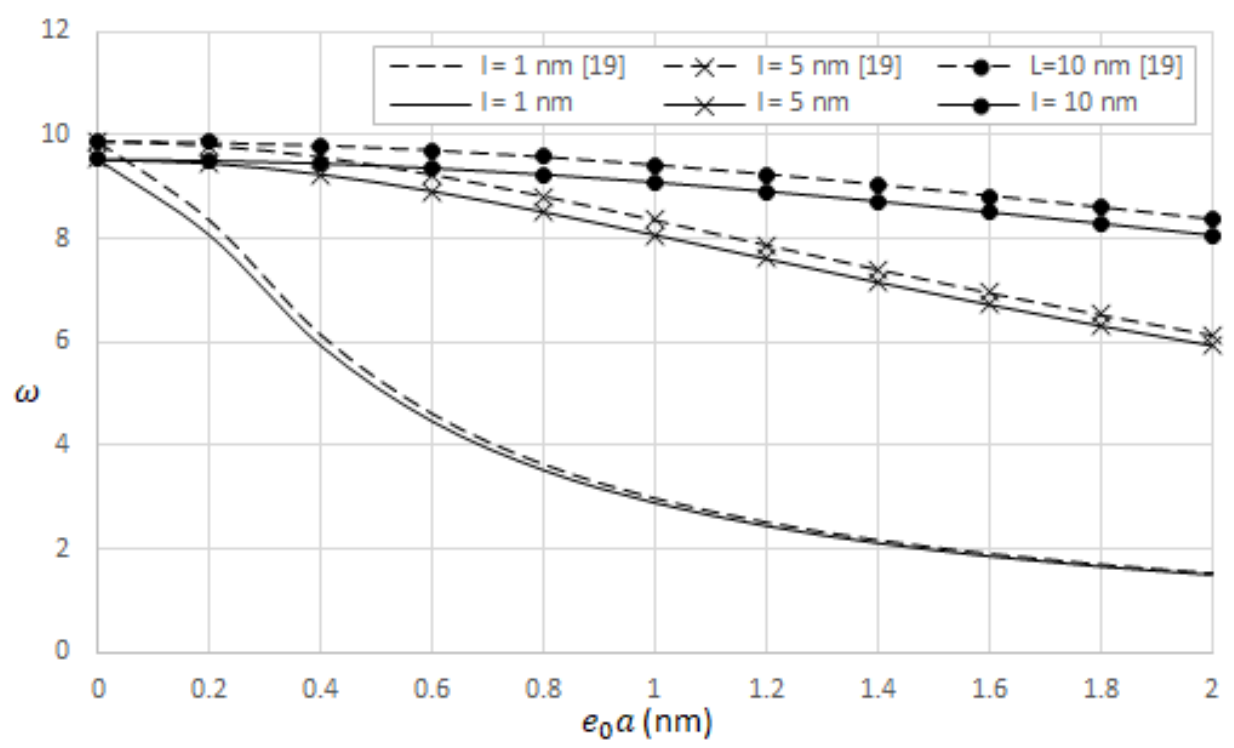

FiguRE 2. Natural frequency $(\omega)$ versus nonlocal parameter $(\eta)$.

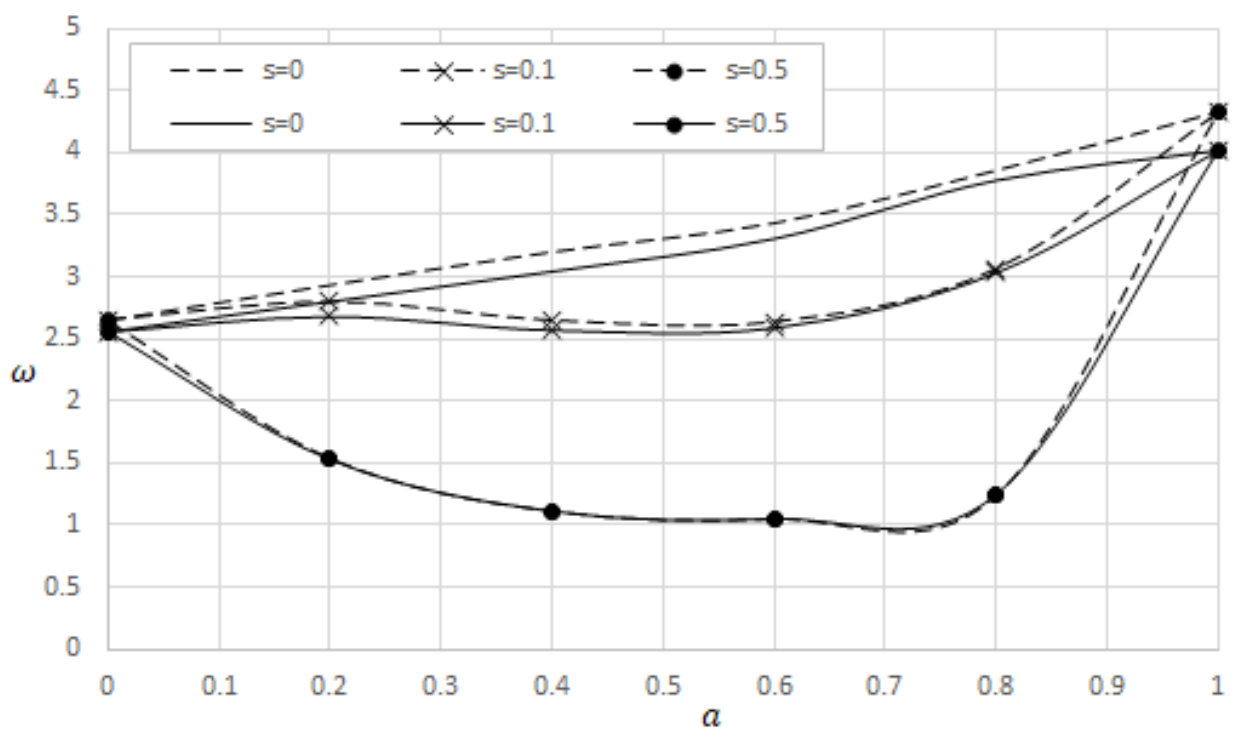

FiguRE 3. Natural frequency $(\omega)$ versus location of crack $(a)$. 


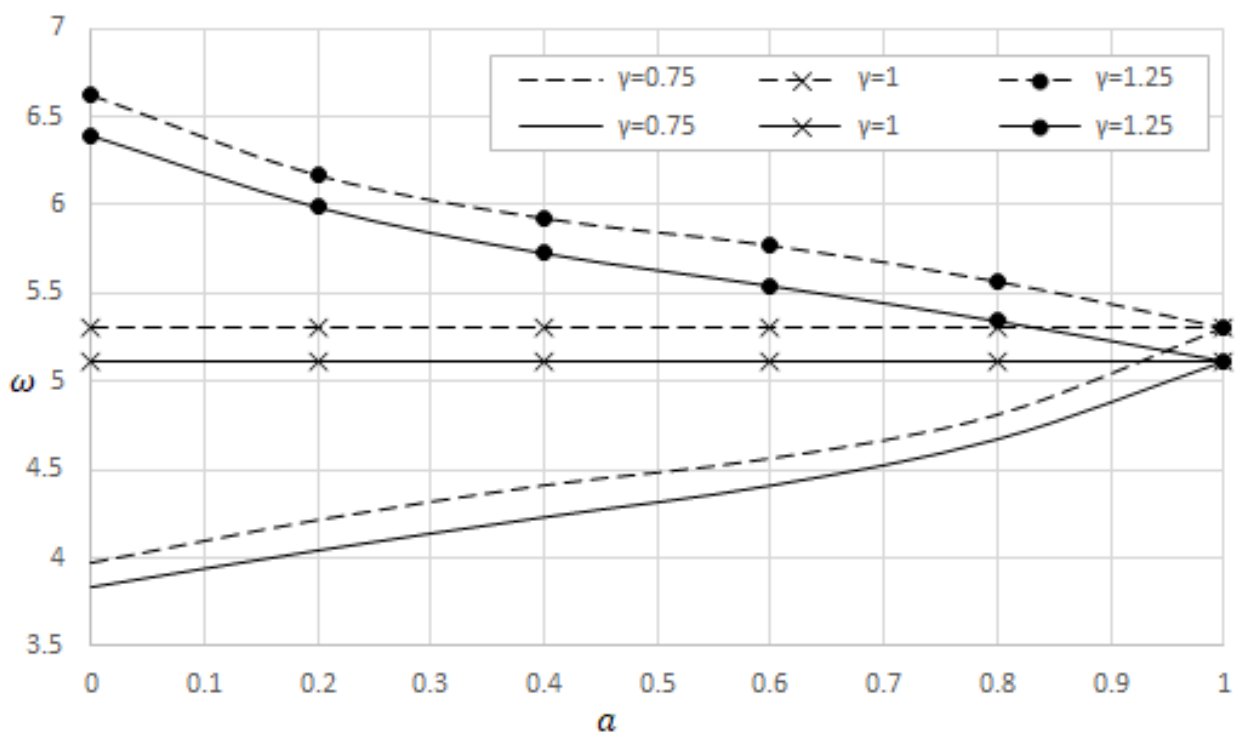

Figure 4. Natural frequency $(\omega)$ versus location of step $(a)$.

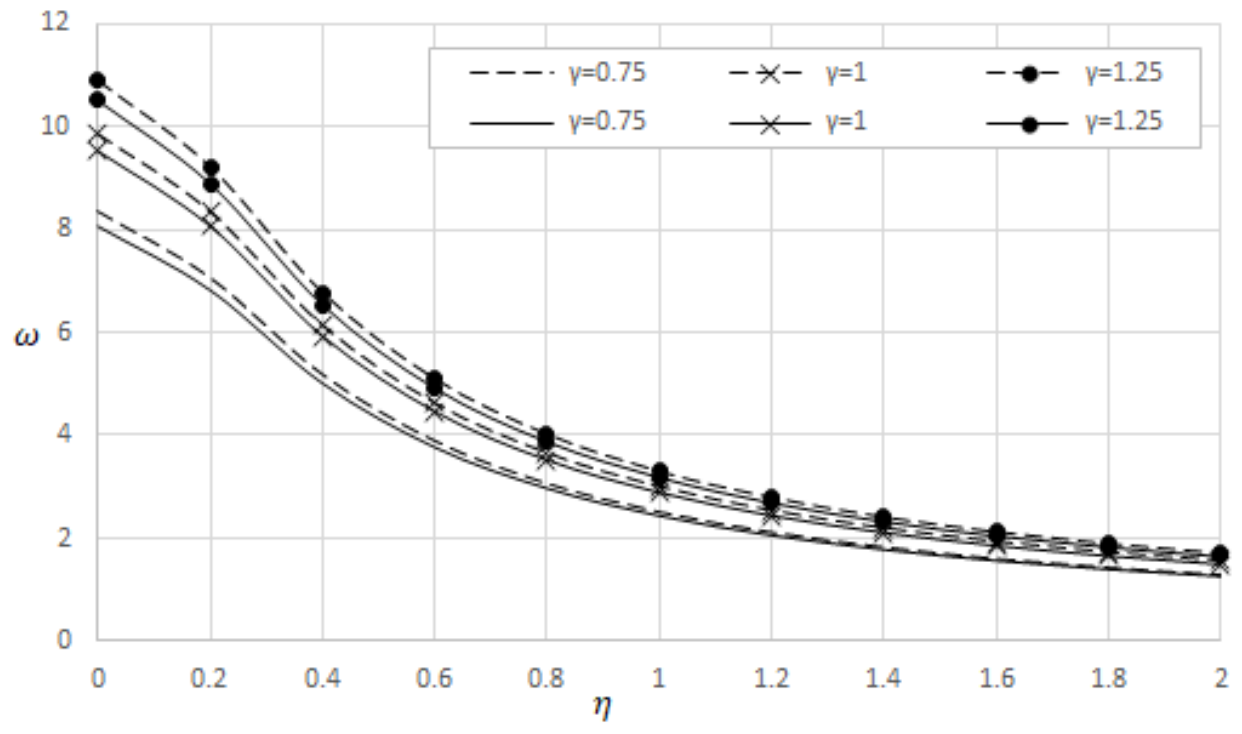

Figure 5. Natural frequency $(\omega)$ versus nonlocal parameter $(\eta)$. 


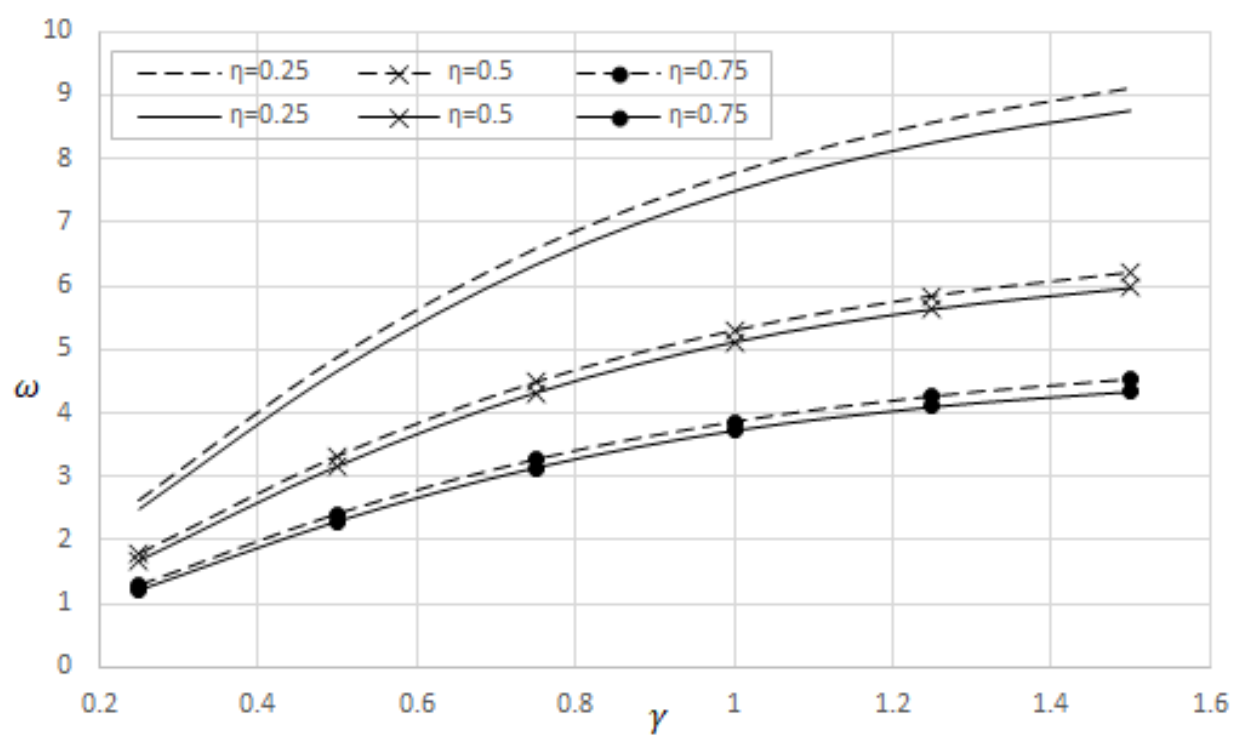

FiguRE 6. Natural frequency $(\omega)$ versus step height ratio $(\gamma)$.

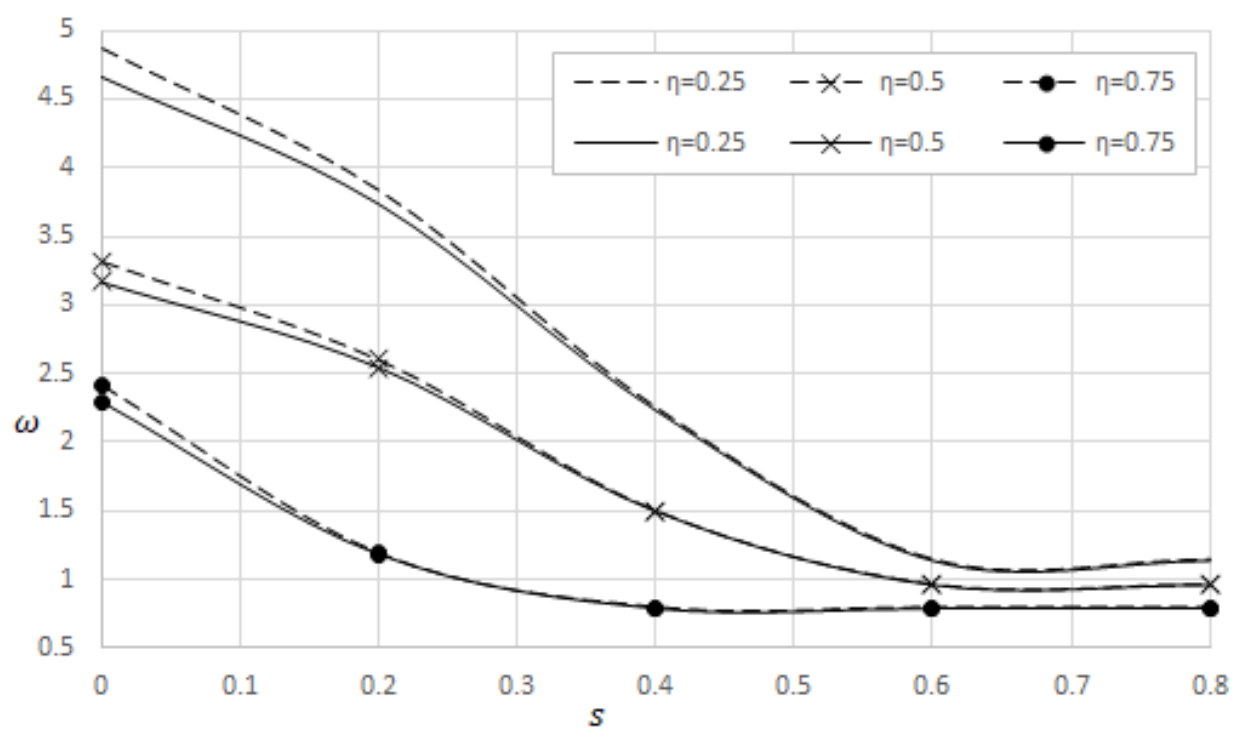

Figure 7. Natural frequency $(\omega)$ versus crack depth ratio $(s)$. 


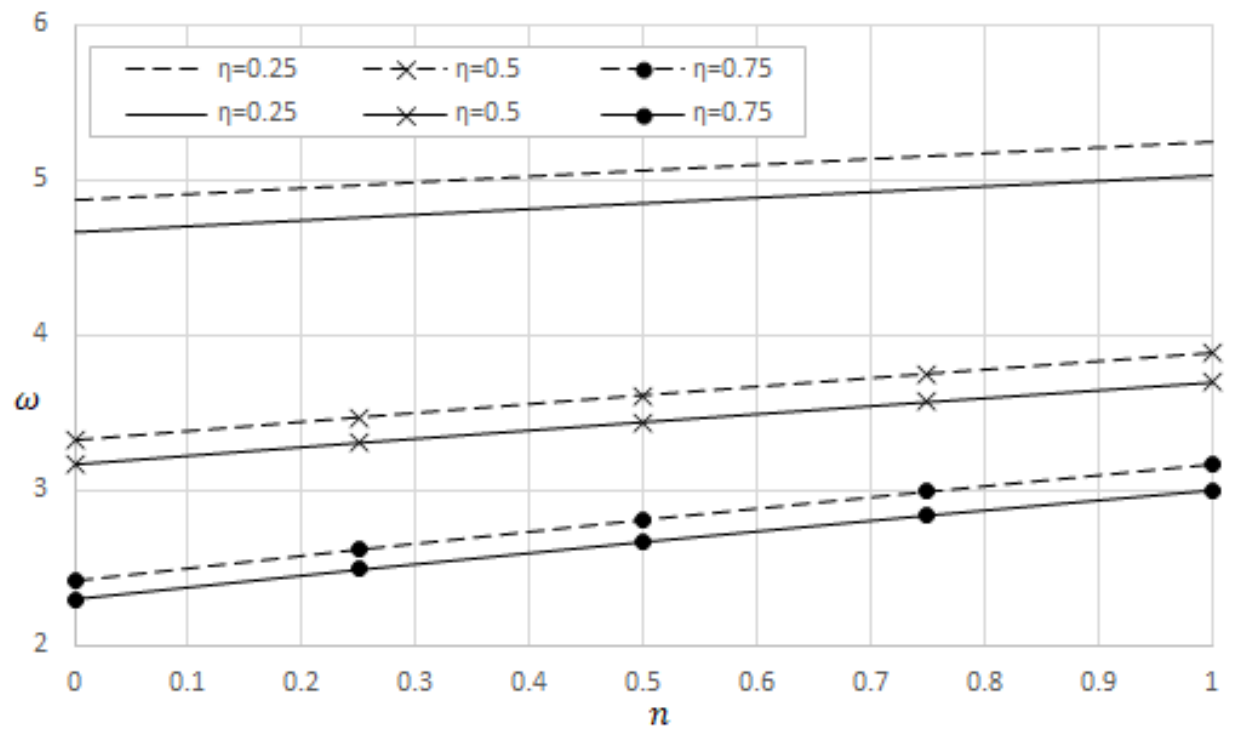

Figure 8. Natural frequency $(\omega)$ versus axial load $(n)$.

In Figure 3, the natural frequency versus step location is presented for different crack sizes. The upper pair of curves corresponds to a nanostrip without cracks; the lower one to the case where the crack has presented half of the thickness. It can be seen from Figure 3 that the location strongly affects the natural frequency.

In Figure 4 the natural frequency versus step location is presented for nanosheets with different step size. In this figure, the natural frequency is depicted in the cases of step height ratios $\gamma=0.75, \gamma=1$ and $\gamma=1.25$, respectively. In calculations the nonlocal parameter $\eta$ is taken equal to 0.5. It can be seen from Figure 4 that the natural frequency increases when $a$ (step location) increases if step height ratio $\gamma=0.75$ (the thickness in the left-hand part of the nanobeam is greater than that in the right-hand part of the nanostrip). However, if the step height ratio $\gamma=1.25$, then the natural frequency decreases when $a$ (step location) increases. This tendency is the same for the theories accounting for the rotatory inertia and for these neglecting this effect (Figure 4).

In Figure 5 the natural frequency versus the nonlocal parameter is presented for different values of the ratio of thicknesses. It can be seen from Figure 5 that the natural frequency decreases with the increase of nonlocal parameter. The results presented in Figure 5 pertain to the nanostrips with the step located at $a=0.5 l$ in the cases where step height ratios 
$\gamma=0.75, \gamma=1$ and $\gamma=1.25$, respectively. The results of the current approach are compared with those of the nonlocal theory neglecting the effect of rotational inertia. It can be seen from Figure 5 that the rotatory inertia has perceivable influence on the natural frequency of stepped nanostrips. However, if the nonlocal parameter $\eta$ tends to the value 2, then both approaches lead to a common value. Figure 5 admits to draw the conclusion that in the case of greater admissible values of the nonlocal parameter $\eta$ the influence of the effect of rotatory inertia is less remarkable.

The natural frequency versus the height ratio is displayed in Figure 6 for different values of a nonlocal parameter. It can be seen from Figure 6 that the natural frequency increases with the increase of the step ratio. The natural frequency versus the ratio of thicknesses $\gamma$ (step height ratio) is portrayed in Figure 6 for fixed values of nonlocal parameter $\eta=0.25$ and $\eta=0.5$, respectively. It can be seen from Figure 6 that both theories (with and without the effect of rotatory inertia) lead to similar results, if step height ratio $\gamma<0.2$. The discrepancy of the results increases, if the ratio of thicknesses increases.

The influence of the crack on the natural frequency of stepped nanostrips is portrayed in Figure 7 for the case where step location $a=0.5 l$ and height (right side) $h_{1}=0.5 h_{0}$. The horizontal axis in Figure 7 shows the nondimensional crack length $s=c / h_{0}$. Figure 7 reveals the matter that for larger values of the crack length different approaches lead to the same result. It can be seen from Figure 7 that if the crack length $c>0.4 h_{0}$ the results obtained by theories accounting for and neglecting, respectively, the rotatory inertia practically coincide.

Figure 8 describes the natural frequency versus axial load for different values of the nonlocal parameter. It can be seen from Figure 8 that the natural frequency increases with the increases of axial load. Here step position $a=0.5 l$ and height (right side) $h_{1}=0.5 h_{0}$. It can be seen from Figure 8 that the natural frequency increases together with the axial load of nanostrips, as might be expected. Note that this effect takes place on the macro level, as well.

\section{Conclusion}

A method for determination of natural frequencies of stepped nanostrips with defects has been developed. In the current paper the equations of vibration of nanostrips accounting for the rotatory inertia of the cross-sections are used. Calculations carried out showed that the crack-like defects essentially affect the natural frequencies of nanostrips. The results of calculations showed that the lowest values of eigenfrequencies correspond to nanostrips having the deepest cracks. The comparison of the results of current work 
with these of earlier works revealed the matter that the theories incorporating the rotatory inertia and these ignoring this effect lead to similar results in the case of nanostrips with large cracks.

\section{Acknowledgements}

The partial support from the Institutional Research Funding IUT2057 of Estonian Ministry of Education and Research is gratefully acknowledged. Also, the support from Estonian Doctoral School in Mathematics and Statistics is acknowledged.

\section{References}

[1] T. L. Anderson, Fracture Mechanics Fundamentals and Applications, Taylor \& Francis Group CRC Press, Boca Raton, 2005.

[2] N. Anifantis and A. Dimarogonas, Post buckling behavior of transverse cracked columns, Comput. Struct. 18(2) (1984), 351-356.

[3] R. Ansari and S. Sahmani,Bending behavior and buckling of nanobeams including surface stress effects corresponding to different beam theories, Internat. J. Engrg. Sci. 49(11) (2011), 1244-1255.

[4] J. Aranda-Ruiz, J. Loya, and J. Fernández-Sáez, Bending vibrations of rotating nonuniform nanocantilevers using the Eringen nonlocal elasticity theory, Comput. Struct. 94(9) (2012), 2990-3001.

[5] M. Aydogdu, A general nonlocal beam theory: its application to nanobeam bending, buckling and vibration, Physica E: Low-Dimensional Systems and Nanostructures 41(9) (2009), 1651-1655.

[6] S. M. Bagdatli, Non-linear vibration of nanobeams with various boundary conditions based on nonlocal elasticity theory, Composites Part B 80 (2015), 43-52.

[7] N. Challamel and C. M. Wang, The small length scale effect for a non-local cantilever beam: A paradox solved, Nanotechnology 19 (2008), 345703.

[8] A. Dimarogonas, Vibration of cracked structures: A state of the art review, Eng. Fracture Mech. 55(5) (1996), 831-857.

[9] A. Dimarogonas, S. Paipetis, and T. Chondros, Analytical Methods in Rotor Dynamics, Springer, Dordrecht, 2013.

[10] A. C. Eringen, Nonlocal Continuum Field Theories, Springer-Verlag, New York, 2002.

[11] A. C. Eringen and D. G. B. Edelen, On nonlocal elasticity, Internat. J. Engrg. Sci. 10(3) (1972), 233-248.

[12] J. Fernández-Sáez, R. Zaera, J. A. Loya, and J. N. Reddy, Bending of Euler-Bernoulli beams using Eringen's integral formulation: A paradox resolved, Internat. J. Engrg. Sci. 99 (2016), 107-116.

[13] S. A. M. Ghannadpour, B. Mohammadi, and J. Fazilati, Bending, buckling and vibration problems of nonlocal Euler beams using Ritz method, Comput. Struct. 96 (2013), $584-589$.

[14] M. Hossain and J. Lellep, Natural vibration of stepped nanoplate with crack on an elastic foundation, in: IOP Conference Series: Materials Science and Engineering 660 (2019), 012051.

[15] J. Lellep and E. Kägo, Vibrations of elastic stretched strips with cracks, Int. J. Mech. 5 (2011), 27-34.

[16] J. Lellep and A. Lenbaum, Free vibrations of stepped nanobeams, Int. J. Comput. Methods Exp. Meas. 6(4) (2018), 716-725. 
[17] J. Lellep and A. Lenbaum, Natural vibrations of stepped nanobeams with defects, Acta Comment. Univ. Tartu. Math. 23(1) (2019), 143-158.

[18] J. Lellep and A. Liyvapuu, Natural vibrations of stepped arches with cracks, Agron. Res. 14(3) (2016), 821-830.

[19] C. Li, C. W. Lim, J. L. Yu, and Q. C. Zeng, Analytical solutions for vibration of simply supported nonlocal nanobeams with an axial force, Int. J. Struct. Stab. Dyn. 11(2) (2011), 257-271.

[20] C. W. Lim, C. Li, and J. L. Yu, The effects of stiffness strengthening nonlocal stress and axial tension on free vibration of cantilever nanobeams, Interaction Multiscale Mech. 2(3) (2009), 223-233.

[21] P. Lu, H. P. Lee, and C. Lu, Dynamic properties of flexural beams using a nonlocal elasticity model, J. Appl. Phys. 99(7) (2006), 073501.

[22] S. M. Mousavi, J. Paavola, and J. N. Reddy, Variational approach to dynamic analysis of third-order shear deformable plates within gradient elasticity, Meccanica 50 (2015), $1537-1550$.

[23] J. Peddieson, G. G. Buchanan, and R. P. McNitt, Application of nonlocal continuum models to nanotechnology, Internat. J. Engrg. Sci. 41(3-5) (2003), 305-312.

[24] C. Polizzotto, Nonlocal elasticity and related variational principles, Int. J. Solids Struct. 38(42-43) (2001), 7359-7380.

[25] J. N. Reddy, Nonlocal theories for bending, buckling and vibration of beams, Internat. J. Engrg. Sci. 45(2-8) (2007), 288-307.

[26] H. Roostai and M. Haghpanahi, Vibration of nanobeams of different boundary conditions with multiple cracks based on nonlocal elasticity theory, Appl. Math. Model. 38(3) (2014), 1159-1169.

[27] W. Soedel, Vibrations of Shells and Plates, M. Dekker CRC Press, New York, 2004.

[28] H. Tada, P. C. Paris, and G. R. Irwin, The Stress Analysis of Cracks Handbook, Saint Louis: Paris Productions, 1985.

[29] H. Thai, A nonlocal beam theory for bending, buckling and vibration of nanobeams, Internat. J. Engrg. Sci. 52 (2012), 56-64.

[30] C. M. Wang, H. Zhang, N. Challamel, and W. H. Duan, On boundary conditions for buckling and vibration of nonlocal beams, Eur. J. Mech. A Solids 61 (2017), 73-81.

[31] C. M. Wang, Y. Y. Zhang, and X. Q. He, Vibration of nonlocal Timoshenko beams, Nanotechnology 18 (2007), 105401.

[32] M. Wesolowski and E. Barkanov, Improving material damping characterization of a laminated plate, J. Sound Vibration 462 (2019), 114928.

[33] S. T. Yaghoubi, S. M. Mousavi, and J. Paavola, Strain and velocity gradient theory for higher-order shear deformable beams, Arch. Appl. Mech. 85 (2015), 877-892.

[34] Y. Y. Zhang, C. M. Wang, and N. Challamel, Bending buckling and vibration of micro/nano-beams by hybrid nonlocal beam model, J. Eng. Mech. ASCE 136(5) (2010), $562-574$.

[35] L. Zhou and Y. Huang, Crack effect on the elastic buckling behavior of axially and eccentrically loaded columns, Struct. Eng. Mech. 22(2) (2006), 169-184.

Institute of Mathematics and Statistics, University of Tartu, Estonia

E-mail address: mainul.hossain@ut.ee

E-mail address: jaan.lellep@ut.ee 